\title{
Direct reconstitution and study of SUN protein interactions in vitro using mammalian cell-free expression
}

Sagardip Majumder ${ }^{1, \#, ~ Y e n-Y u ~ H s u ¹, ~ M i c h a e l ~ A n d r e a s ², ~ T o b i a s ~ W . ~ G i e s s e n ², 3, ~ A l l e n ~ P . ~ L i u ~}{ }^{1,2,4,5^{*}}$

1 Department of Mechanical Engineering, University of Michigan, Ann Arbor, Michigan, 48109, USA

${ }^{2}$ Department of Biomedical Engineering, University of Michigan, Ann Arbor, Michigan, 48109, USA

${ }^{3}$ Department of Biological Chemistry, University of Michigan, Ann Arbor, Michigan, 48109, USA

${ }^{4}$ Cellular and Molecular Biology Program, University of Michigan Medical School, Ann Arbor, Michigan, 48109, USA

${ }^{5}$ Department of Biophysics, University of Michigan, Ann Arbor, Michigan, 48109, USA

\# Present address: Institute of Protein Design, University of Washington, Seattle, Washington, 98195,

USA

${ }^{*}$ Corresponding author: Allen P. Liu

Email: allenliu@umich.edu

Author Contributions: S.M and A.P.L conceived the project and designed experiments. S.M and Y.Y.H carried out the experiments and prepared figures. M.A. and T.W.G designed and performed the Cryo-EM experiment. S.M analyzed relevant data. S.M and A.P.L wrote the manuscript.

Competing Interest Statement: The authors declare no competing interests.

Keywords: Membrane protein reconstitution; SUN-KASH interaction; mammalian cell-free expression

This PDF file includes:

Main Text

Figures 1 to 4

Supplemental Figures 1 to 5 and Table S1 
bioRxiv preprint doi: https://doi.org/10.1101/2021.04.11.439350; this version posted August 24, 2021. The copyright holder for this preprint (which was not certified by peer review) is the author/funder. All rights reserved. No reuse allowed without permission.

\begin{abstract}
SUN proteins are an integral part of LINC (Linker of Nucleoskeleton and Cytoskeleton) complex which spans the nuclear envelope and acts as a physical tether between the cytoskeletal filaments and the nuclear lamina. Several human diseases associated with nuclear deformation are primarily caused by impaired functioning of SUN proteins. Studies in yeast and mammalian cells have illustrated the detrimental effects of different SUN mutants in nuclear positioning and movement. While cell-based studies provide physiological relevance to the functioning of a protein, in vitro reconstitution of isolated proteins is useful in mechanistically dissecting protein function in a biochemically defined environment. In this study, we used a mammalian cell-free expression system to synthesize and reconstitute SUN proteins in artificial lipid bilayer membranes. Building on our previous work demonstrating directional reconstitution of full-length SUN proteins, we deciphered the mechanism of such protein reconstitution and leveraged it to test several theories/models of LINC complex assembly. By using a simple fluorescence-based assay, we revealed the importance of cations such as calcium and the presence of disulfide bonds in the formation of LINC complexes. Through sequential reconstitutions of SUN proteins and soluble luminal domains of SUN proteins, we found that coiled coil domains of SUN proteins are necessary for homomeric and heteromeric interactions of reconstituted SUN proteins. Overall, our results provide mechanistic insights on LINC complex formation and how this might impact cellular mechanotransduction. The facile approach for reconstituting full-length membrane proteins can be extended to investigate other difficult-to-study membrane proteins in vitro.
\end{abstract}




\section{Introduction}

As a highly regulated membrane barrier that surrounds the genome-containing nucleus in eukaryotic cells, nuclear envelope (NE) plays a critical role in segregating the nucleus from the cytoplasmic environment for chromatin organization and gene regulation. In addition to molecular transport across the NE through nuclear pore complexes, the nucleus is physically tethered to the different components of the cytoskeleton to sense and transmit mechanical forces through the NE. The linker of nucleoskeleton and cytoskeleton (LINC) complex formed between the outer nuclear membrane (ONM) proteins containing Klarsicht, ANC-1, and Syne homology (KASH) domain, and Sad1 and UNC84 (SUN) homology domain proteins anchored to the inner nuclear membrane (INM), are evolutionarily conserved NE bridges essential for nuclear integrity and migration, chromosome movement and pairing during meiosis and nuclear mechanotransduction (1). Studies involving the soluble domains of KASH and SUN proteins exposed to the perinuclear space have revealed important structural features of SUN$\mathrm{KASH}$ interactions within the LINC complex $(2,3)$. Fig. 1A illustrates a schematic of a SUN protein trimer linked to KASH proteins to form the LINC complex. Cell-based studies have identified the different interacting domains of LINC complex proteins and their localization signals that result in their transport to the respective membranes of the $N E(1,4,5)$. However, predictions based on interactions between soluble fragments of the LINC complex proteins are only useful when their effects can be studied with full-length proteins embedded in lipid bilayer membranes. Unfortunately, transient or long-term SUN protein expression in cells do not serve this purpose well as many redundant pathways can mediate SUN-KASH binding which makes it difficult to study native interactions. In this regard, in vitro reconstitution of LINC complex proteins enables the investigation of membrane-constrained proteinprotein interactions in the absence of any regulatory pathways (6).

In our previous work, we developed a simple bottom-up synthetic biology platform based on a mammalian cell-free expression (CFE) system for the rapid reconstitution and study of LINC complex assembly using full-length SUN proteins (7). The most abundant isoforms of SUN proteins (SUN1 and SUN2) from mouse were expressed in the presence of supported lipid bilayers with excess membrane reservoirs (SUPER) templates (8) to mediate spontaneous reconstitution. By carrying out protease protection assay on these artificial nuclear membranes (ANMs), we found evidence of directional insertion of N-terminally tagged full-length SUN1 and SUN2 (EGFP-SUN1 ${ }^{\mathrm{FL}}$ and EGFP-SUN2 ${ }^{\mathrm{FL}}$ ) with their $\mathrm{C}$ termini facing the solvent accessible side of the membranes. We also predicted the number of transmembrane domains of both SUN1 and SUN2 on the basis of association of different SUN truncation mutants with SUPER templates. Further, we reconstituted partial LINC complex assembly through interaction of reconstituted SUN proteins with synthetically generated peptides mimicking the KASH domain of ONM protein nesprin-2 from mouse. Given these initial successes, we next utilize the developed platform to explore and examine some previously predicted but unverified aspects of SUN$\mathrm{KASH}$ interactions.

The formation of LINC complex depends on the oligomeric states of SUN proteins as the trimeric configuration of SUN domain facilitates binding to KASH domain proteins $(3,9)$. Coiled coil domains in SUN proteins are predicted to regulate trimerization by switching from an inactive to active state while simultaneously stabilizing a cation loop coordinated with potassium for efficient KASH binding (10, 11). Past studies showed that even in the absence of SUN1 which is crucial for meiotic processes, meiotic recombination events and telomere attachment and movement within the nuclear envelope is functional as SUN2 co-localizes with KASH5 at the sites of telomere attachment (12). This suggests possible interaction and complementarity in function between SUN1 and SUN2. On the other hand, competition between SUN1 and SUN2 leading to the formation of LINC complexes that selectively bind to microtubule or actin filaments plays a crucial role in regulating nuclear anchorage and movement (13). Elevated levels of SUN1 in progeria patient fibroblasts were associated with hindered nuclear movement which was rescued in cells with native proportions of SUN1 and SUN2 (13). Intracellular levels of $\mathrm{Ca}^{2+}$ can also affect monomer-trimer transition of SUN proteins. Recent structural data suggests that the binding of $\mathrm{Ca}^{2+}$ to coiled coil domains potentially activates trimerization of SUN2 thereby triggering KASH binding (14). In view of these findings, we sought to test these ideas using an in vitro setup partly because of the complexity of probing SUN biology in cells. In this work, we have extended our established platform to test these predictions and designed simple assays for real-time detection. We first explore the mechanism of SUN protein reconstitution in ANMs followed by investigating the effect of calcium and redox state on LINC complex formation. Finally, we leverage the modularity of our CFE system to reconstitute full-length wild type SUN proteins and study homomeric and heteromeric interactions between their solvent accessible domains.

\section{Results}




\section{SUN proteins localize to native microsome-like structures when expressed in HeLa cell-free system.}

We have previously investigated the reconstitution of full-length SUN proteins in the supported lipid bilayer membranes of SUPER templates (7). SUPER templates are supported lipid bilayers on silica beads that provide a suitable substrate for incorporation of membrane spanning proteins with the added advantage of their handling and imaging like cells since they can be easily pelleted and washed. In our previous study, we observed directional insertion of both SUN1 and SUN2 with each retaining the ability to bind to KASH peptides in vitro. A key advantage of the system was the directional reconstitution in artificial membranes in the absence of additional modifications to the CFE system. This led us to explore the mechanism of SUN protein synthesis and its subsequent localization to the SUPER templates.

A consistent observation in lysates obtained through mechanical lysis is the presence of cell membrane fragments. Past studies with CFE systems derived from Chinese hamster ovary (CHO) cells have demonstrated the presence of endoplasmic reticulum (ER) fragments called microsomes in the cell lysate which are translationally active and aid in localization of ER-secreting proteins to the microsomal membranes (15-17). The use of ER microsomes in in vitro translation systems is a standard biochemical technique which was revolutionary in the discovery of translocons and membrane protein chaperones in eukaryotic cells (18-20). However, the proteins translated in such reactions were primarily ER-secreting proteins such as prolactin with known localization signaling sequences (21-23). SUN proteins are known to have multiple regulatory elements at their $\mathrm{N}$-termini which result in nuclear transport and localization to the INM $(24,25)$. So, it is possible that a mammalian CFE system made from HeLa cells could have ER fragments that can recognize and translocate SUN proteins. Fig. 1B shows confocal images of bulk CFE reactions expressing EGFP-SUN1 ${ }^{\mathrm{FL}}$ and EGFP-SUN2 ${ }^{\mathrm{FL}}$ stained with a lipophilic stain Dil. We observed co-localization of EGFP SUN proteins and Dil in the puncta indicating the association of SUN proteins with membranous structures in bulk.

Using a standard ultracentrifugation workflow as indicated in Fig. 1C, we spun down CFE reactions with saturated yields (i.e., reactions that reached completion) of both SUN1 and SUN2 and tested their presence in equal volumes of supernatant and pellet fractions (see Methods for details). Since the volume of CFE reactions was small $(\sim 10 \mu \mathrm{l})$, no significant pellet was visible. Staining of the CFE lysate with an ER tracker dye revealed dense punctate structures in the lysate but absent in the supernatant following ultracentrifugation (Fig. 1D). Based on these observations we asked if a fraction of the cell-free expressed SUN proteins could associate with the microsomes. We observed higher concentrations of both SUN proteins in the pellet fractions compared to the supernatant fractions (Fig. 1E). A negative control with cell-free expressed EGFP had the same protein concentration in the two fractions. Further, expression of a bacterial channel protein MscL (mechanosensitive channel of large conductance) that is a capable of self-insertion into lipid membranes (26-28) was enriched in the pellet fraction as well. The presence of microsomal structures in lysates is further supported by the cryo-EM image shown in Fig. 1F. Lipid bilayer vesicles in the size range of 50-200 nm were clearly visible in our homemade CFE system, although their surface was not as densely covered with ribosomes as has been shown with cryoelectron tomography (29). These results indicate possible translocation of SUN proteins in mammalian microsomes.

\section{Fusion between microsomes and supported lipid bilayer enables directional reconstitution of cell-free expressed SUN proteins.}

To confirm membrane insertion of SUN proteins in microsomes, we carried out a protease digestion assay followed by in-gel fluorescence measurement. Correct folding and insertion of SUN1 and SUN2 would require the proteins to span the bilayer with each terminus on opposite sides of the membrane. Given our N-terminally tagged EGFP constructs, we were able to probe for EGFP signal post protease digestion. If the nucleoplasmic domain $\left(S U N^{N D}\right)$ is exposed to the solvent upon CFE synthesis, one would expect the EGFP to be degraded by the addition of protease. Fig. 2A shows ingel fluorescence images of full-length or luminal domains (SUN ${ }^{L D}$ ) of cell-free expressed SUN1 and SUN2 exposed to protease digestion. Bands at approximately $50+\mathrm{kDa}$ indicate the protection of the nucleoplasmic domains of the full-length proteins in bulk CFE reactions. In contrast, SUN ${ }^{L D}$ proteins were completely degraded following protease exposure. Therefore, it is likely that a fraction of wild type SUN proteins, in the absence of signaling sequences, are translocated to the microsomes in our HeLa CFE system.

To test the hypothesis that SUN protein reconstitution on supported lipid bilayer is mediated via microsome fusion instead of direct reconstitution, we used SUPER template as our model supported lipid bilayer. SUPER templates are formed by using a high ionic strength buffer to form a loosely bound bilayer membranes which provide larger surface area and sufficient substrate-bilayer spacing to 
accommodate membrane proteins with large soluble domains (Fig. 2B). Previously, fluorescencebased quantification demonstrated the presence of excess membrane on SUPER templates that minimizes the effects of substrate interaction (8). Thus, SUPER templates serve as a superior model membrane system compared to the tightly bound supported lipid bilayers for SUN protein. Additionally, to partially mimic inner nuclear membrane composition, we used a combination of lipids found in yeast nucleus with added cholesterol $(7,30)$.

We next asked how the topology of SUN protein insertion into the ANMs is unidirectional given their translocation to microsomes in CFE reactions. One plausible hypothesis is spontaneous fusion of microsomal membranes with the lipid membranes of SUPER templates. Past studies have demonstrated the role played by cations and lipid charges in spontaneous vesicle fusion specially between liposomes with high curvature and planar lipid bilayers $(31,32)$. Since CFE reactions contain $\mathrm{mM}$ levels of potassium and magnesium ions and the SUPER templates are made with charged lipids (phosphatidylserine) and lipids that mediate spontaneous fusion at room temperature (phosphatidylethanolamine) $(33,34)$, the mechanics of membrane fusion can ensure consistent topology of reconstituted integral membrane proteins as opposed to direct membrane insertion. In order to probe this mechanism of SUN protein transport to the ANMs, we compared ANM localization of EGFP-SUN1 ${ }^{\mathrm{FL}}$ and EGFP-SUN2 ${ }^{\mathrm{FL}}$ under two conditions. Fig. $2 \mathrm{C}$ i) depicts the default scenario where SUPER templates are incubated with CFE reactions expressing the SUN constructs right from the start of the reactions. In the second scheme, depicted in Fig. $2 \mathrm{C}$ ii), the SUPER templates are added to the CFE reactions after proteins have been synthesized. If the SUN proteins are co-translationally inserted into ANMs, then one might expect a significant reduction in EGFP-SUN intensity on SUPER templates for case ii) compared to case i). However as shown in Fig. 2D, we observed similar association of both EGFP-SUN1 ${ }^{\mathrm{FL}}$ and EGFP-SUN2 ${ }^{\mathrm{FL}}$ with the SUPER templates under the two different cases mentioned above. This indicates that reconstitution of SUN proteins in ANMs can be carried out by addition of saturated CFE reactions to SUPER templates. While direct post-translational insertion of SUN proteins in these membranes can explain the lack of difference between the two cases, it is unlikely that SUN proteins with large soluble domains and multi-membrane associating domains can self-insert into synthetic lipid bilayers while maintaining specific membrane topology. Vesicle fusion on the other hand is the most plausible mechanism of protein reconstitution here.

We hypothesize that adding SUPER templates to the CFE reactions at the start of protein synthesis, as opposed to after protein synthesis is completed, should make no difference to the orientation of SUN proteins in the ANMs as the mechanism of protein transfer is through microsome fusion. This hypothesis is supported by the Western blot shown in Fig. 2E where SUPER templates incubated with CFE following the protocol shown in Fig. 2C ii) were subjected to washes with a chaotropic agent (6 M urea) to distinguish between transient vs strong interactions of SUN proteins in ANM membranes. For weakly associated or membrane adsorbed proteins, urea wash is expected to disrupt such interactions $(35,36)$, resulting in a lack of bands in the membrane fraction of SUPER templates. However, all membrane associating SUN mutants (N-terminally tagged with EGFP) as studied in our previous work (7) showed strong interaction with ANM membranes as evident from the bands observed under the membrane fraction column (Fig. 2E). The supernatant fraction in this case corresponds to the bulk CFE reactions that were added to the SUPER templates and shows saturated levels of indicated SUN proteins. Finally, for bulk CFE reactions, the pellet fraction following Airfuge centrifugation contains (see methods) SUN proteins and can be reconstituted on SUPER templates, as demonstrated with SUN1 (Fig. 2F). By comparison, a soluble protein is not enriched in the pellet fraction following the same assay (Fig. S3). Altogether, the HeLa CFE system enables translocation of fulllength and tagged SUN proteins in microsomal membranes; and in the presence of SUPER templates leads to directional reconstitution potentially through vesicle fusion.

\section{Cation loop and disulfide bonds play crucial role in SUN-KASH interactions in vitro.}

Generation of ANMs with EGFP-SUN1 ${ }^{\mathrm{FL}}$ and EGFP-SUN2 ${ }^{\mathrm{FL}}$ leads to the exposure of the Ctermini of both the proteins to the solvent accessible side of the membrane (7). This protein orientation was leveraged in our previous study to demonstrate the binding of a synthetic KASH peptide to both SUN1 and SUN2. To our knowledge, it was the first demonstration of SUN-KASH interactions in vitro with full-length proteins embedded in a synthetic lipid bilayer.

Past studies primarily used soluble mutants of SUN proteins which were purified and their interaction with $\mathrm{KASH}$ peptides analyzed through biochemical and structural techniques. Current structural models of the SUN-KASH complex have suggested the presence of cation loops in the canonical SUN trimeric state and that the KASH lid is closely associated with the SUN domains through disulfide bonds (10). Though such structural data have been obtained for different SUN-KASH pairs from soluble proteins, these interactions are difficult to study in cells especially since the LINC complex 
spans the perinuclear space. Thus, our platform is well suited for testing SUN-KASH interactions under different biochemical conditions in vitro that may not be easy to test in cells.

Previous models indicate the size of the cation loop to perfectly fit a potassium ion for each SUN monomer (3). However, since the CFE reactions we used had mM levels of potassium, probing the effect of potassium on SUN-KASH interactions was not feasible. Instead, we looked at the role played by calcium ions in mediating KASH binding because of negligible levels of calcium in our homemade CFE mixture $(37,38)$. TRITC-KASH ${ }^{\mathrm{WT}}$ and TRITC-KASH ${ }^{\mathrm{dPPPT}}$ peptides were incubated with reconstituted EGFP-SUN1 ${ }^{\mathrm{FL}}$ and EGFP-SUN2 ${ }^{\mathrm{FL}}$ both in the presence and absence of $1 \mathrm{mM}$ calcium. Fig. 3A shows EGFP and TRITC fluorescence images of EGFP-SUN1 ${ }^{\mathrm{FL}}$ interaction with both KASH peptides with or without calcium. The corresponding quantification plot in Fig. 3B indicates increased TRITC intensities for the case where the SUPER templates were exposed to $1 \mathrm{mM}$ calcium during $\mathrm{KASH}$ binding. It is important to note that the same batch of SUPER templates was distributed equally to carry out the respective KASH binding assays. Similar observations were obtained for the interaction of the same KASH peptides with EGFP-SUN2 ${ }^{\mathrm{FL}}$ (Fig. 3C and 3D). While previously unreported, these observations indicate that calcium ions promote SUN-KASH interactions.

Using a similar approach, we next looked at the effect of the redox state of SUN proteins in mediating $\mathrm{KASH}$ binding in vitro. Conserved cysteines in SUN proteins form disulfide bonds when interacting with the PPPX motif of KASH domain containing proteins like nesprins (39). Thus, we investigated the effect of high concentrations of a strong reducing agent like dithiothreitol (DTT) on SUN-KASH interactions. Following the KASH binding experiment with TRITC-KASH ${ }^{\mathrm{WT}}$, SUPER templates with reconstituted SUN proteins were exposed to $10 \mathrm{mM}$ DTT. The images shown in Fig. 3E provide evidence of weakened KASH binding for both SUN proteins within 15 minutes of DTT addition. Quantification of TRITC fluorescence (Fig. 3F) reveals a four-fold decrease in KASH binding upon exposure to DTT in a timespan of 15 minutes. No significant decrease in EGFP fluorescence was found at the end of this experiment.

\section{Sequential SUN protein reconstitution reveals the importance of coiled coil domains in mediating SUN-SUN homomeric and heteromeric interactions.}

One of the characteristic features of LINC complexes is the formation of oligomeric SUN-KASH interacting structures across the nuclear envelope. Recent models of LINC complex formation propose a transition between an inactive and active state for both SUN1 and SUN2 proteins based on the spatial orientation of their coiled coil domains $(14,40)$. Both SUN proteins have two coiled coil domains (CC1 and CC2) spaced by a few amino acids (Fig. 1A) and their roles in mediating SUN heterooligomerization is not entirely understood. SUN2-KASH binding is primarily trimeric for different types of KASH proteins (10) whereas higher oligomeric interactions of SUN1-KASH have been proposed previously (14). Since we were able to reconstitute full-length SUN proteins bound to KASH peptides in synthetic membranes, we asked whether the membrane-embedded proteins could form oligomeric structures through interactions between their soluble domains. Given the versatility of CFE systems, we expressed SUN1 ${ }^{\mathrm{FL}}$ and SUN2 ${ }^{\mathrm{FL}}$ with added GreenLysine-tRNA (GreenLys) which enabled fluorescence detection of membrane incorporated wild type proteins. Using this strategy, ANMs with both SUN1 ${ }^{\mathrm{FL}}$ and SUN2 ${ }^{\mathrm{FL}}$ labelled with fluorescent lysine can be localized to the SUPER templates similar to the EGFP-tagged constructs (Fig. 4A).

We designed an assay to investigate the interaction between reconstituted wild type SUN proteins and their soluble counterparts (i.e. luminal domains). Fig. 4B shows an experimental scheme where the same SUPER templates were exposed to two rounds of CFE reactions expressing different proteins with washes in between. In the first round, unlabeled full-length SUN proteins (SUN ${ }^{\mathrm{FL}}$ ) were reconstituted on SUPER templates which were then treated with a second round of CFE expressing either EGFP or EGFP-SUN ${ }^{\mathrm{LD}}$. Expressing EGFP-SUN ${ }^{\mathrm{LD}}$ without expressing SUN ${ }^{\mathrm{FL}}$ in CFE-1 served as a control (shown as a schematic bound by dashed lines in Fig. 4B). As shown in Fig. 1A, SUN corresponds to the SUN mutant with luminal domain only and does not associate with the SUPER templates (7). Therefore, any EGFP fluorescence detected on the SUPER templates after the second round of CFE with SUN ${ }^{\mathrm{LD}}$ indicates interaction between the reconstituted SUN ${ }^{\mathrm{FL}}$ and its soluble luminal domain mutant. Additionally, given two independent rounds of CFE, it is possible to expose $S U N 1^{\mathrm{FL}}$ to EGFP-SUN2 ${ }^{\mathrm{LD}}$ and SUN2 ${ }^{\mathrm{FL}}$ to EGFP-SUN1 ${ }^{\mathrm{LD}}$ in order to study heteromeric SUN protein interactions. As coiled coil domains are crucial to the formation of SUN oligomers during KASH binding, the effect of coiled coil deletions on SUN-SUN interactions could also be studied using this scheme. Therefore, we created deletion mutants for both SUN proteins in which either or both the coiled coil domains were deleted (SUN $\left.{ }^{\mathrm{DEL}} \mathrm{CC} 1, \mathrm{SUN}^{\mathrm{DEL}} \mathrm{CC} 2, \mathrm{SUN}^{\mathrm{DEL}} \mathrm{CC} 1 \& 2\right)$.

We carried out all combinations of the two CFE reactions with wild type and luminal domain mutants that lacked CC1, CC2 or both CC1 and CC2 (Fig. 4C). We found that EGFP fluorescence from 
the second round of CFE reactions expressing EGFP-SUN ${ }^{L D}$ constructs only localized on the SUPER templates when wild type SUN1 or SUN2 proteins were reconstituted in the first round. CFE of EGFP alone in the second round served as a control experiment indicating there was no non-specific interaction of EGFP with the ANM (Fig. S4). Hence, for reconstituted SUN1 ${ }^{\mathrm{FL}}$ and SUN2 ${ }^{\mathrm{FL}}$, we detected robust homomeric and heteromeric interactions between their luminal domains. In the case of the coiled coil deletion mutants, no significant fluorescence was observed for all cases indicating a lack of homomeric and heteromeric interactions between these SUN mutants and the EGFP-tagged luminal domains of SUN1 and SUN2. Membrane association and incorporation of each of the coiled coil deletion mutants was tested using labelled lysine as mentioned before (Fig. S5). Altogether, our findings suggest that deletion of either coiled coil domains from SUN1 or SUN2 is sufficient to disrupt oligomeric interactions between SUN proteins.

\section{Discussion}

In this work, we demonstrate the use of a CFE system for in vitro reconstitution to study SUN protein interactions with full-length proteins in synthetic membranes. We first investigated the mechanism of directional protein reconstitution in SUPER templates as evidenced from our previous study and leveraged the specific SUN protein topology in the ANMs to study SUN-KASH and SUN-SUN interactions. The use of full-length SUN1 and SUN2 with fused EGFP or incorporated GreenLys and their reconstitution on $5 \mu \mathrm{m}$ diameter beads enabled direct detection of protein localization and subsequent interactions by using fluorescence microscopy. With a bead-based platform such as the SUPER templates, it was possible to design simple biochemical assays to probe protein function owing to the ease of handling reconstituted SUN proteins. The major advantage of a CFE system is its modularity in synthesizing many different proteins with minimal alterations $(41,42)$. As a result, SUNSUN interactions could be probed through multiple rounds of CFE on the same batch of SUPER templates while expressing different mutants. Another aspect of this platform is the ability to simultaneously synthesize and translocate SUN proteins into the synthetic membranes of SUPER templates. While the C-terminally exposed topology of SUN1 and SUN2 in the ANMs was a discovery and not a controlled reconstitution, the evidence of microsome-based delivery of SUN proteins through membrane fusion provides a way to possibly control protein orientation in SUPER templates by cotranslational modification of SUN constructs with additional transmembrane domains as used previously $(43,44)$. SUPER templates serve as an ideal membrane model in this study as opposed to standard supported lipid bilayers owing to presence of excess membrane reservoir not tightly bound to the bead substrate (8). This is reflected in the binding of reconstituted SUN1 and SUN2 to TRITC-KASH'WT peptide which should only be possible if SUN proteins oligomerize in the ANMs. Further, observation of small puncta on the SUPER templates could be evidence of SUN clustering in these excess membrane reservoirs suggesting sufficient protein mobility which would be difficult to achieve in conventional supported lipid bilayers (45). The short timescale for the entire reconstitution step with sufficient yield of proteins to mediate fluorescence-based detection is a key feature which enabled us to carry out the different studies presented here.

Our results only provide a window to the range of possibilities with such a platform. For instance, one could look at the effect of different concentrations of calcium on KASH binding to wild type SUN proteins. The specific cysteine residues responsible for disulfide bond formation during KASH binding (39) can be mutated and their effect studied without significant changes to the demonstrated approach. Further insights can be gained by quantitative estimation of the timescale of DTT-based dissociation of the LINC complex. Similar extensions of the current work can be carried out to further investigate the possibility of hybrid LINC complexes based on SUN1-SUN2 interactions. Finally, the entire approach can be directly applied to the study of LINC complex formation between SUN1 and SUN2 and other types of KASH peptides. Overall, our findings provide strong support to some of the existing hypothesis about LINC complex formation while new insights were gained with respect to the importance of certain interactions in SUN-KASH binding. Specifically, we found that calcium ions significantly enhance the ability of reconstituted SUN proteins to bind to KASH peptides but are not necessary for LINC complex formation. Since ER is contiguous with the NE membrane, ER calcium signaling elicited by mechanical signal (46) can strengthen SUN-nesprin interaction thus priming mechanotransduction. On the other hand, disulfide bonds are indispensable for KASH binding with both SUN1 and SUN2. Similarly, ER redox state that is closely linked to ER protein-folding homeostasis (47) may also be connected to regulation of SUN-nesprin interactions. Finally, it is likely that SUN oligomerization cannot be achieved in the absence of either coiled coil domains for both the SUN proteins. In summary, we developed a modular platform for the study of in vitro reconstituted SUN proteins with an emphasis on real-time detection through fluorescence microscopy and a possibility to use simple biochemical techniques to 
bioRxiv preprint doi: https://doi.org/10.1101/2021.04.11.439350; this version posted August 24, 2021. The copyright holder for this preprint (which was not certified by peer review) is the author/funder. All rights reserved. No reuse allowed without permission.

probe SUN biology. Such a platform can be extended to study interactions of other difficult-to-study membrane proteins. 


\begin{abstract}
Methods
Materials.

Chemicals. All lipids were purchased from Avanti Polar Lipids. Other reagents used have been previously described (7). Dithiothreitol (DTT) was purchased from Sigma-Aldrich. GreenLysine-tRNA (GreenLys) was purchased from Promega.

Plasmids. All EGFP tagged and untagged full-length mouse SUN protein plasmids were gifted to us by Dr. G.W.G. Luxton (University of California, Davis) and were used in our previous study (7). All coiled coil mutants were generated from untagged wildtype SUN1 and SUN2 constructs by mutagenesis (NEB site directed mutagenesis kit) (Table S1).
\end{abstract}

Preparation of SUPER templates. Supported lipid bilayer with excess membrane reservoir (SUPER) templated beads were made following a previously published protocol (44) and our prior work with SUN proteins (7). ANM composition consists of 45\% DOPC, $27 \%$ DOPE, $9 \%$ DOPS, $2.2 \%$ phosphatidic acid, and $16.8 \%$ cholesterol.

CFE of SUN proteins. A homemade HeLa CFE system was made based on previously published protocols $(35,36)$. A typical reaction volume of $10 \mu$ l was assembled with $5 \mathrm{nM}$ plasmid in a $1.5 \mathrm{~mL}$ eppendorf tube and incubated at $30^{\circ} \mathrm{C}$ for up to 3 hours. Most reactions reached saturation after 2 hours. For the standard reconstitution of SUN proteins (Fig. 2C i)), SUPER templates were added to the CFE reaction prior to incubation. When SUPER templates were added to completed CFE reactions, the mixture was incubated at room temperature for approximately 15 minutes with intermittent shaking prior to subsequent analysis. All reconstituted SUPER templates were washed with 2 mI PBS or with 6 $M$ urea as indicated. For fluorescent visualization of non-EGFP-tagged proteins, GreenLys was added to the CFE reaction at a dilution of 1:50 as recommended in the manufacturer's protocol.

Ultracentrifugation with Airfuge. A Beckman Coulter Airfuge was used to spin down CFE reactions. Completed CFE reactions were collected in a 1.5-mL microcentrifuge tube and then mixed with $30 \mu \mathrm{l}$ of extraction buffer (20 mM HEPES- $\mathrm{KOH}, \mathrm{pH} 7.5,45 \mathrm{mM}$ potassium acetate, $45 \mathrm{mM} \mathrm{KCl,} 1.8 \mathrm{mM}$ magnesium acetate, $1 \mathrm{mM}$ dithiothreitol (DTT)). $40 \mu \mathrm{l}$ of mixture was then transferred to an ultracentrifuge tube and centrifuged at around $100,000 \mathrm{~g}$ for 15 minutes at room temperature using the Airfuge (Beckman Coulter). After the centrifugation, $20 \mu \mathrm{l}$ of the supernatant was carefully recovered and transferred to a $1.5-\mathrm{mL}$ microcentrifuge tube without disturbing the pellet, and the remaining $20 \mu \mathrm{l}$ of pellet fraction was resuspended by pipetting up and down for mixing thoroughly before transferring to another microcentrifuge tube. To investigate the protein incorporation, $2 \mu$ of SUPER templates were added and incubated with the supernatant and pellet fractions respectively for 30 minutes and then centrifuged at $300 \mathrm{~g}$ for 3 minutes. SUPER templates were visible as a small white pellet which was gently resuspended by tapping for downstream analysis.

Protease digestion assay. Pronase (Roche Cat No.: 10165921001) was dissolved in DI (de-ionized) water at a stock concentration of $10 \mathrm{mg} / \mathrm{ml}$. $2 \mu \mathrm{l}$ of this stock was added to $8 \mu \mathrm{l}$ of bulk CFE reaction and incubated at room temperature for 15-20 minutes. Thereafter $2 \mu \mathrm{l}$ of $25 \mathrm{X}$ Complete mini protease inhibitor cocktail (Millipore-Sigma Cat No.: 11836153001) was added to the digested CFE and incubated for 10 minutes. This mixture was then subsequently analyzed with SDS-PAGE or Western blot.

KASH binding assay. Reconstituted SUN proteins on SUPER templates were washed with PBS. The beads were resuspended in $30 \mu \mathrm{l}$ PBS and distributed into $9 \mu$ laliquots in eppendorf tubes. To two aliquots, $\sim 300 \mathrm{nM}$ TRITC-KASH ${ }^{\mathrm{WT}}$ was added followed by addition of $1 \mathrm{mM}$ calcium chloride (final conc.) in one and an equal volume of DI water in another. To the third aliquot, $2300 \mathrm{nM}$ of TRITC$\mathrm{KASH}^{\mathrm{dPPPT}}$ was added followed by dilution with calcium chloride to a final concentration of $1 \mathrm{mM}$. The three tubes were then incubated at room temperature for 10 minutes before subsequent imaging. In order to investigate the effect of DTT, 10 mM DTT (final conc.) was added after incubation of the TRITC$\mathrm{KASH}$ to SUN protein-reconstituted SUPER templates.

In-gel imaging and Western blots. In-gel imaging of SUN proteins was carried out on a Sapphire biomolecular imager (Azure Biosystems) for both EGFP tagged and GreenLysine-containing proteins. Samples were loaded on 4-20\% gradient bis-Tris PAGE gels (Bio-Rad) with 4X Laemmeli loading buffer (Bio-Rad). Samples were not heated to retain in-gel EGFP and GreenLysine fluorescence. Rabbit AntiGFP antibody (Abcam) was used to detect EGFP tagged SUN mutants. 
Confocal fluorescence microscopy and analysis. Confocal images of SUPER templates were taken as previously described on a spinning disk confocal microscope and subsequent analysis was carried out using ImageJ as before (7). Briefly, 5-10 line scans for each bead for the relevant scenario were taken and the peak intensities recorded. Subsequently, their mean was taken and combined with intensity values from $10-15$ beads for background correction and statistical analysis. Pairwise student $t$-tests and one factor ANOVA for the plots obtained in Fig. 3 were carried out from background subtracted intensity values obtained using ImageJ. The values were then normalized by the maximum intensity of the group before plotting.

Grid preparation. CFE reactions expressing EGFP-SUN1 ${ }^{\mathrm{FL}}$ were ultracentrifuged as mentioned above and $3.5 \mu \mathrm{L}$ of the pellet fraction was applied to glow discharged grids (Quantifoil R1.2/1.3 Cu 200 mesh, glow discharged 1 minute, $5 \mathrm{~mA}$ ) and frozen by plunging into liquid ethane using an FEI Vitrobot Mark IV (100\% humidity, temperature: $22^{\circ} \mathrm{C}$ or $4^{\circ} \mathrm{C}$, blot force: 20 , blot time: 4 seconds, wait time: 0 seconds). Grids were clipped and stored in liquid nitrogen until analyzed.

Cryo-Electron Microscopy (Cryo-EM). Cryo-EM movies were collected using a Thermo Fisher Scientific Glacios cryo-electron microscope operating at 200 kV equipped with a Gatan K2 Summit direct electron detector at the University of Michigan Life Sciences Institute. Movies were collected at $45,000 x$ magnification (pixel size $-0.980 \AA$ ) or $22,000 x$ magnification (pixel size - $2.005 \AA$ ) using Leginon software package with exposure time of 8 seconds, frame time of $200 \mathrm{~ms}$, and total dose of $63 \mathrm{e}^{-/} \AA^{2}$ (48). Movies were collected at defocus of either $-3.00 \mu \mathrm{m}$ or $-1.80 \mu \mathrm{m}$. Representative still images from movies were obtained using the Leginon software package.

\section{Acknowledgments}

The authors thank G.W. Gant Luxton (University of California, Davis) for his feedback and helpful discussions regarding some of the experiments.

\section{Funding sources}

This research was supported by NIH Grants R21GM134167 and R01EB030031 and NSF Grant 1935265 to A.P.L. T.W.G. acknowledges support from NIH R35GM133325. 


\section{Reference:}

1. M. Crisp, et al., Coupling of the nucleus and cytoplasm: Role of the LINC complex. J. Cell Biol. (2006) https:/doi.org/10.1083/jcb.200509124.

2. B. A. Sosa, U. Kutay, T. U. Schwartz, Structural insights into LINC complexes. Curr. Opin. Struct. Biol. 23 (2013).

3. B. A. Sosa, A. Rothballer, U. Kutay, T. U. Schwartz, LINC Complexes Form by Binding of Three KASH Peptides to Domain Interfaces of Trimeric SUN Proteins. Cell 149 (2012).

4. C. Ostlund, et al., Dynamics and molecular interactions of linker of nucleoskeleton and cytoskeleton (LINC) complex proteins. J. Cell Sci. 122 (2009).

5. Q. Wang, X. Du, Z. Cai, M. I. Greene, Characterization of the Structures Involved in Localization of the SUN Proteins to the Nuclear Envelope and the Centrosome. DNA Cell Biol. 25 (2006).

6. H. Jia, P. Schwille, Bottom-up synthetic biology: reconstitution in space and time. Curr. Opin. Biotechnol. 60 (2019).

7. S. Majumder, P. T. Willey, M. S. DeNies, A. P. Liu, G. Luxton, A synthetic biology platform for the reconstitution and mechanistic dissection of LINC complex assembly. J. Cell Sci. 132, jcs219451 (2019).

8. S. Neumann, T. J. Pucadyil, S. L. Schmid, Analyzing membrane remodeling and fission using supported bilayers with excess membrane reservoir. Nat. Protoc. (2013) https:/doi.org/10.1038/nprot.2012.152.

9. W. Wang, et al., Structural insights into SUN-KASH complexes across the nuclear envelope. Cell Res. 22 (2012).

10. V. E. Cruz, F. Esra Demircioglu, T. U. Schwartz, Structural Analysis of Different LINC Complexes Reveals Distinct Binding Modes. J. Mol. Biol. 432 (2020).

11. D. I. Kim, B. Kc, K. J. Roux, Making the LINC: SUN and KASH protein interactions. Biol. Chem. 396, 295-310 (2015).

12. J. Link, et al., Analysis of Meiosis in SUN1 Deficient Mice Reveals a Distinct Role of SUN2 in Mammalian Meiotic LINC Complex Formation and Function. PLoS Genet. 10 (2014).

13. W. Chang, et al., Imbalanced nucleocytoskeletal connections create common polarity defects in progeria and physiological aging. Proc. Natl. Acad. Sci. 116 (2019).

14. Z. Jahed, et al., Molecular Insights into the Mechanisms of SUN1 Oligomerization in the Nuclear Envelope. Biophys. J. 114 (2018).

15. L. Thoring, S. Kubick, "Versatile Cell-Free Protein Synthesis Systems Based on Chinese Hamster Ovary Cells" in (2018) https:/doi.org/10.1007/978-1-4939-8730-6_19.

16. A. Zemella, T. Richter, L. Thoring, S. Kubick, "A Combined Cell-Free Protein Synthesis and Fluorescence-Based Approach to Investigate GPCR Binding Properties" in (2019) https:/doi.org/10.1007/978-1-4939-9121-1_4.

17. S. K. Dondapati, et al., Functional Reconstitution of Membrane Proteins Derived From Eukaryotic Cell-Free Systems. Front. Pharmacol. 10 (2019).

18. F. Lyko, B. Martoglio, B. Jungnickel, T. A. Rapoport, B. Dobberstein, Signal Sequence Processing in Rough Microsomes. J. Biol. Chem. 270 (1995).

19. B. Jungnickel, T. A. Rapoport, A posttargeting signal sequence recognition event in the endoplasmic reticulum membrane. Cell 82 (1995).

20. E. Carlson, N. Bays, L. David, W. R. Skach, "Reticulocyte Lysate as a Model System to Study Endoplasmic Reticulum Membrane Protein Degradation" in Ubiquitin-Proteasome Protocols, (Humana Press) https:/doi.org/10.1385/1-59259-895-1:185.

21. M. Sagstetter, R. Zimmermann, Assembly of M13 and M13am8H1R1 procoat protein into microsomes is stimulated by rabbit reticulocyte lysate and ATP. Biochem. Biophys. Res. Commun. 153 (1988).

22. K. Ota, M. Sakaguchi, G. von Heijne, N. Hamasaki, K. Mihara, Forced Transmembrane Orientation of Hydrophilic Polypeptide Segments in Multispanning Membrane Proteins. Mol. Cell 2 (1998).

23. A. Zemella, et al., Cell-free protein synthesis as a novel tool for directed glycoengineering of active erythropoietin. Sci. Rep. 8 (2018).

24. C. P. Lusk, G. Blobel, M. C. King, Highway to the inner nuclear membrane: rules for the road. Nat. Rev. Mol. Cell Biol. 8 (2007).

25. E. C. Tapley, D. A. Starr, Connecting the nucleus to the cytoskeleton by SUN-KASH bridges across the nuclear envelope. Curr. Opin. Cell Biol. 25 (2013).

26. A. R. Battle, E. Petrov, P. Pal, B. Martinac, Rapid and improved reconstitution of bacterial mechanosensitive ion channel proteins MscS and MscL into liposomes using a modified 
sucrose method. FEBS Lett. 583 (2009).

27. M. L. Jacobs, M. A. Boyd, N. P. Kamat, Diblock copolymers enhance folding of a mechanosensitive membrane protein during cell-free expression. Proc. Natl. Acad. Sci. U. S. A. 116, 4031-4036 (2019).

28. S. Majumder, et al., Cell-sized mechanosensitive and biosensing compartment programmed with DNA. Chem. Commun. 53, 7349-7352 (2017).

29. S. Pfeffer, et al., Structure and 3D Arrangement of Endoplasmic Reticulum MembraneAssociated Ribosomes. Structure 20 (2012).

30. G. van Meer, D. R. Voelker, G. W. Feigenson, Membrane lipids: where they are and how they behave. Nat. Rev. Mol. Cell Biol. 9 (2008).

31. J. Bentz, N. Duezguenes, Fusogenic capacities of divalent cations and effect of liposome size. Biochemistry 24 (1985).

32. D. Papahadjopoulos, S. Nir, N. D zg nes, Molecular mechanisms of calcium-induced membrane fusion. J. Bioenerg. Biomembr. 22 (1990).

33. T. M. Allen, K. Hong, D. Papahadjopoulos, Membrane contact, fusion and hexagonal (HII) transitions in phosphatidylethanolamine liposomes. Biochemistry 29 (1990).

34. J. Wilschut, N. Duezguenes, D. Papahadjopoulos, Calcium/magnesium specificity in membrane fusion: kinetics of aggregation and fusion of phosphatidylserine vesicles and the role of bilayer curvature. Biochemistry 20 (1981).

35. X. Yuan, et al., Subcellular localization and membrane association of SARS-CoV 3a protein. Virus Res. 109 (2005).

36. A. LOHIA, A. N. CHATTERJEE, J. DAS, Lysis of Vibrio cholerae Cells: Direct Isolation of the Outer Membrane from Whole Cells by Treatment with Urea. Microbiology 130 (1984).

37. K. K. Y. Ho, J. W. Lee, G. Durand, S. Majumder, A. P. Liu, Protein aggregation with poly(vinyl) alcohol surfactant reduces double emulsion-encapsulated mammalian cell-free expression. PLoS One 12, e0174689 (2017).

38. K. K. Y. Ho, V. L. Murray, A. P. Liu, "Engineering artificial cells by combining HeLa-based cellfree expression and ultrathin double emulsion template" in (2015) https:/doi.org/10.1016/bs.mcb.2015.01.014.

39. N. E. Cain, et al., Conserved SUN-KASH Interfaces Mediate LINC Complex-Dependent Nuclear Movement and Positioning. Curr. Biol. 28 (2018).

40. S. Nie, et al., Coiled-Coil Domains of SUN Proteins as Intrinsic Dynamic Regulators. Structure 24 (2016).

41. J. G. Perez, J. C. Stark, M. C. Jewett, Cell-Free Synthetic Biology: Engineering Beyond the Cell. Cold Spring Harb. Perspect. Biol. 8 (2016).

42. V. Noireaux, A. P. Liu, The New Age of Cell-Free Biology. Annu. Rev. Biomed. Eng. 22, 51-77 (2020).

43. F. Cymer, G. von Heijne, Cotranslational folding of membrane proteins probed by arrestpeptide-mediated force measurements. Proc. Natl. Acad. Sci. 110 (2013).

44. F. Cymer, N. Ismail, G. von Heijne, Weak pulling forces exerted on $\mathrm{N}_{\text {in }}$-orientated transmembrane segments during co-translational insertion into the inner membrane of Escherichia coli. FEBS Lett. 588 (2014).

45. S. Mashaghi, A. M. van Oijen, A versatile approach to the generation of fluid supported lipid bilayers and its applications. Biotechnol. Bioeng. 111 (2014).

46. T.-J. Kim, et al., Distinct mechanisms regulating mechanical force-induced $\mathrm{Ca} 2+$ signals at the plasma membrane and the ER in human MSCs. Elife 4 (2015).

47. S. S. Cao, R. J. Kaufman, Endoplasmic Reticulum Stress and Oxidative Stress in Cell Fate Decision and Human Disease. Antioxid. Redox Signal. 21 (2014).

48. C. Suloway, et al., Automated molecular microscopy: The new Leginon system. J. Struct. Biol. 151, 41-60 (2005). 
A

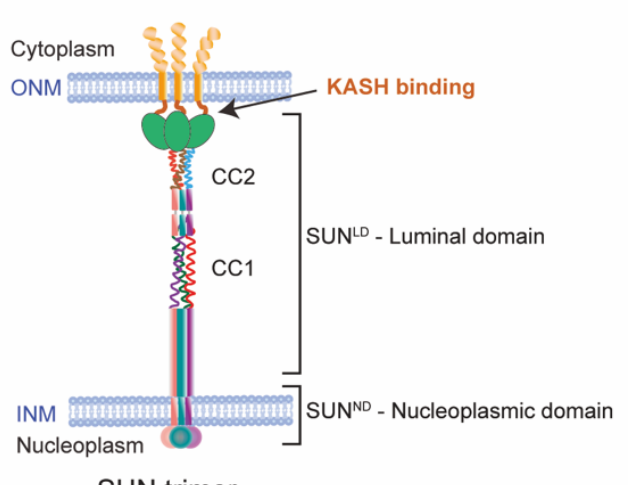

SUN trimer

C

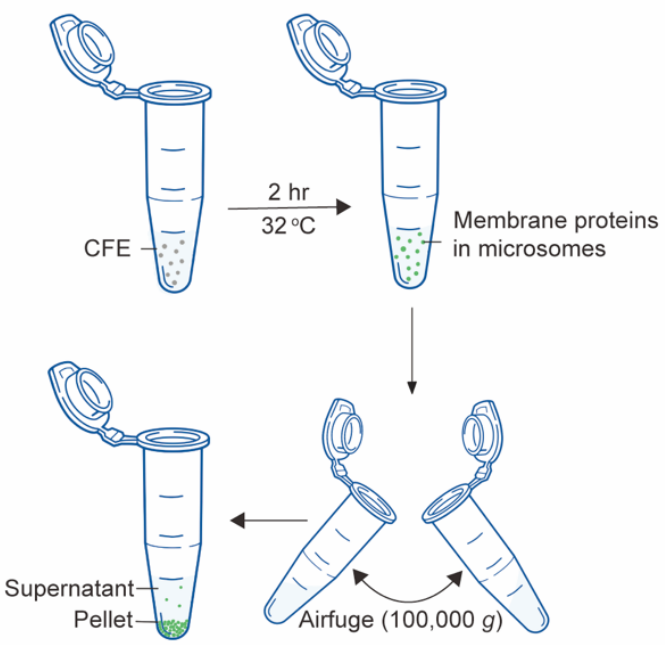

B

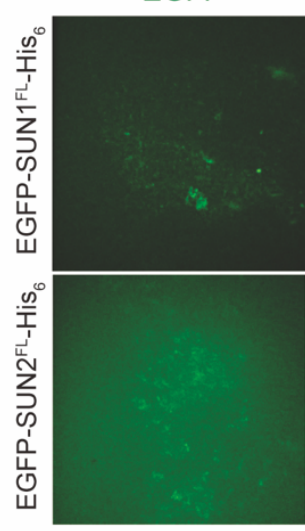

D

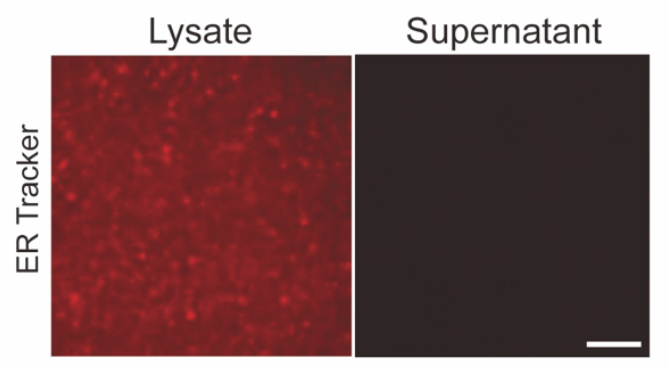

$\mathrm{F}$

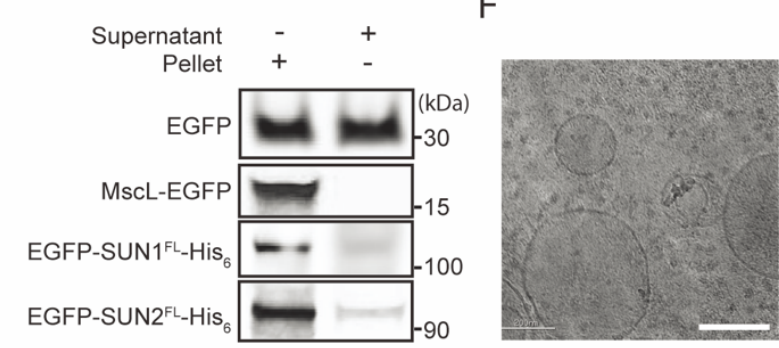

Figure 1. Microsomal structures and association of SUN proteins with membrane fragments in HeLa CFE. A. Cartoon representation of a SUN protein in the inner nuclear membrane with the luminal domain (LD) spanning the perinuclear space and the nucleoplasmic domain (ND) pointing into the nucleus. Typical trimeric configuration is shown that is known to interact with nesprins via KASH binding domains. INM - inner nuclear membrane, ONM - outer nuclear membrane, CC1 \& CC2 - coiled coil domains of SUN proteins. B. Puncta observed in confocal fluorescence images of cell-free expressed SUN proteins in bulk reactions close to the coverslip. Dil staining was used to indicate lipophilic structures in CFE reactions. Merged images shown on right. Scale bar: $5 \mu \mathrm{m}$. C. Schematic illustrating the use of Airfuge to spin down microsomal membranes in bulk CFE reactions. D. Confocal fluorescence images of ER tracker labelled HeLa lysate before and after spinning down microsomal membranes. Scale bar: $1 \mu \mathrm{m}$. E. In-gel fluorescence images of EGFP and SUN proteins in supernatant and pellet fractions post ultracentrifugation. A self-inserting mechanosensitive membrane channel MscL was also expressed and served as a positive control. F. Cryo-EM image of HeLa CFE system expressing EGFP-SUN1 ${ }^{\mathrm{FL}}$ showing vesicular structures. Small structures are visible in the background indicating ribosomes $(\sim 30 \mathrm{~nm})$. Scale bar: $100 \mathrm{~nm}$. Full gel images are shown in Fig. S1. 
A

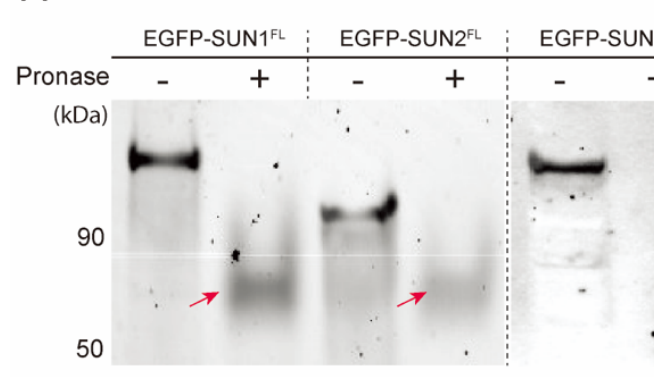

C

i) CFE addition at $0 \mathrm{hr}$

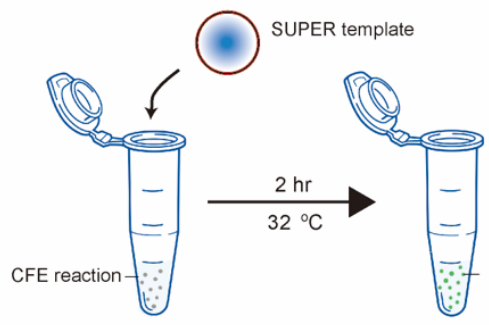

ii) CFE addition at $2 \mathrm{hr}$

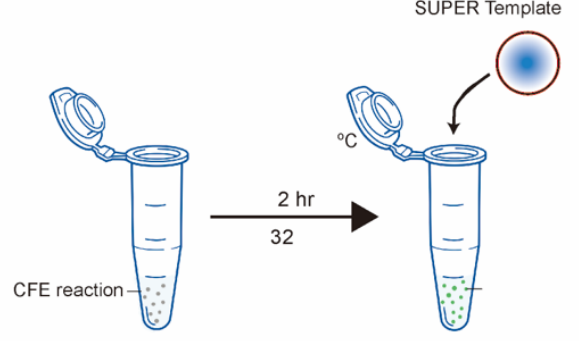

B

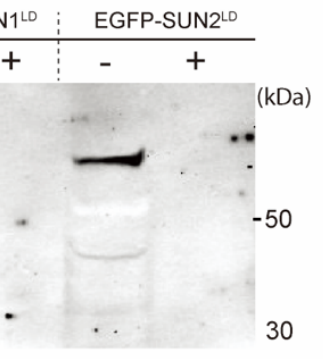

$\mathrm{D}$
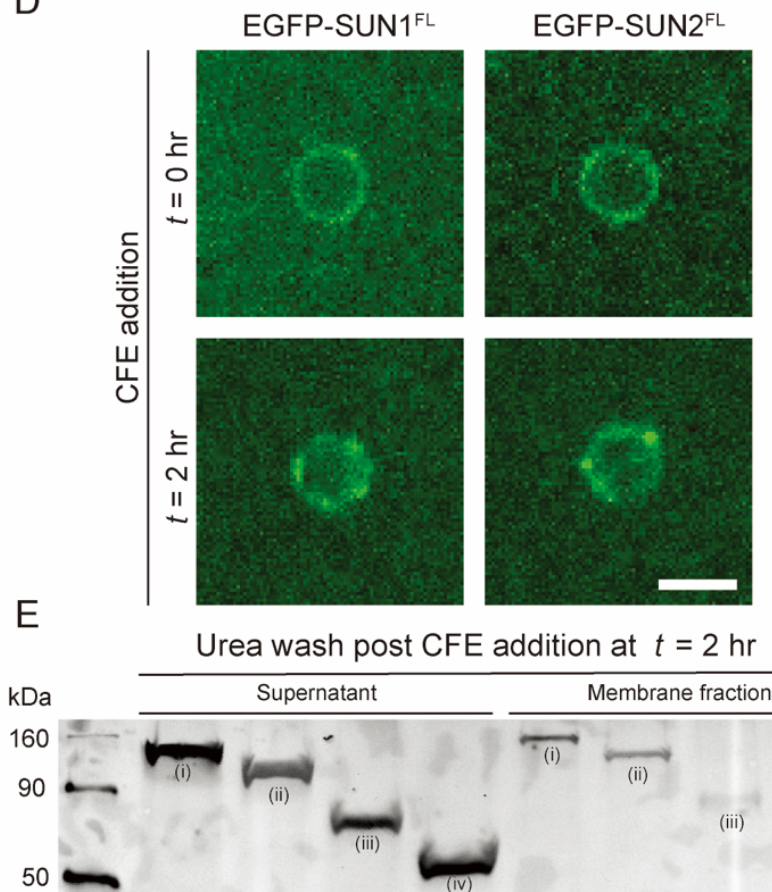

Urea wash post CFE addition at $t=2 \mathrm{hr}$

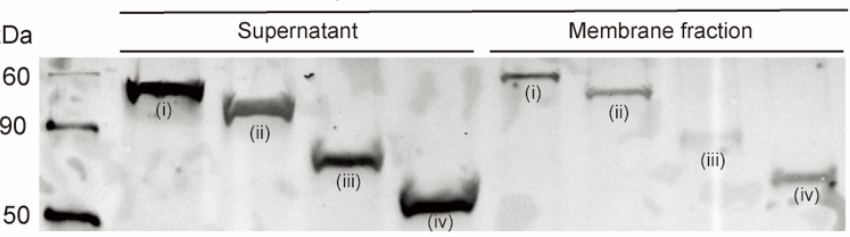

(i) EGFP-SUN1 ${ }^{\mathrm{FL}}$

(iii) EGFP-SUN1 ${ }^{\mathrm{ND}}$

(ii) EGFP-SUN2 ${ }^{\mathrm{FL}}$

(iv) EGFP-SUN2 ${ }^{\mathrm{ND}}$

$\mathrm{F}$

Reconstitution post CFE and centrifugation
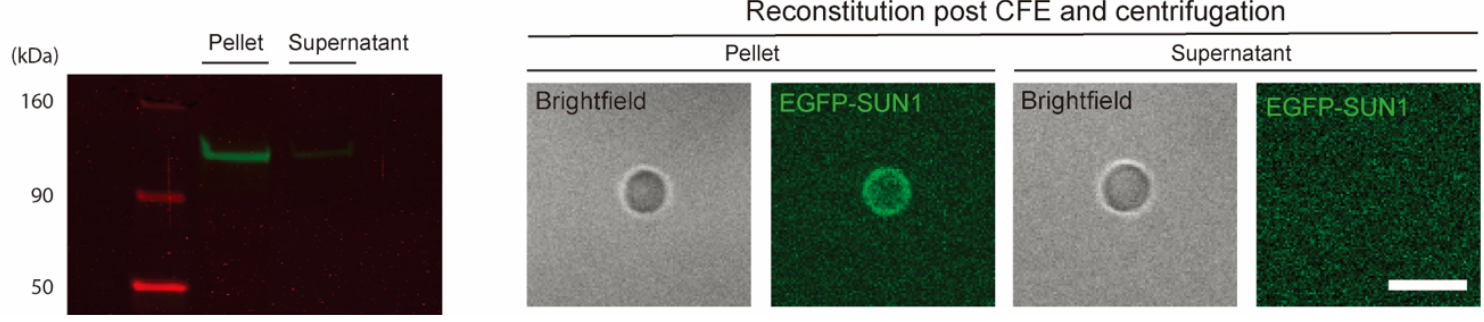

Figure 2. Incorporation of SUN proteins in microsomes and possible fusion-based mechanism of membrane protein reconstitution on supported lipid bilayers. A. In-gel fluorescence from protease digestion of CFE of full-length SUN1 and SUN2 and their luminal domains tagged with EGFP at the $\mathrm{N}$-termini. Arrows indicate bands with incompletely digested proteins observed in the case of fulllength SUN proteins. B. Schematic depicting formation of supported lipid bilayers on silica beads in the presence of low and high ionic strength buffers. As shown, SUPER templates are typically formed when fusion of small vesicles result in the formation of loosely bound unilamellar membranes. C. Test of membrane protein reconstitution on SUPER templates added to CFE of SUN proteins - i) at the onset of CFE reaction or ii) after completion of protein synthesis. D. Confocal fluorescence images of SUPER templates incubated with cell-free expressed SUN proteins for the two cases mentioned in C.. Beads were washed with $6 \mathrm{M}$ urea before imaging. $\mathrm{E}$. Western blot of full-length and nucleoplasmic domains of SUN1 and SUN2 tagged with EGFP at the N-termini added to SUPER templated after $2 \mathrm{hr}$. Supernatant refers to the bulk CFE reactions extracted after incubation with SUPER templates as per protocol ii) in C. while membrane fraction indicates membrane-incorporated proteins in SUPER templates washed with $6 \mathrm{M}$ urea. F. In-gel fluorescent image of pellet and supernatant fractions of a 
bioRxiv preprint doi: https://doi.org/10.1101/2021.04.11.439350; this version posted August 24, 2021. The copyright holder for this preprint (which was not certified by peer review) is the author/funder. All rights reserved. No reuse allowed without permission.

CFE reaction with SUN1-EGFP. The pellet and supernatant fractions were isolated using the Airfuge fractionation assay and subsequently added to SUPER templates for imaging (right) or directly run on gel (left). Scale bars: $5 \mu \mathrm{m}$. Full gel images are shown in Fig. S2. 
A
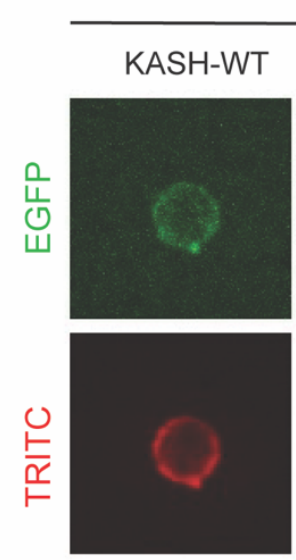

C
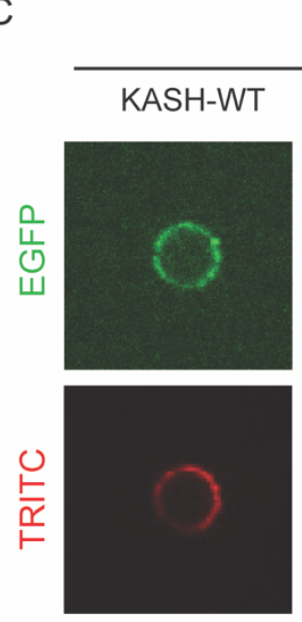

$E$

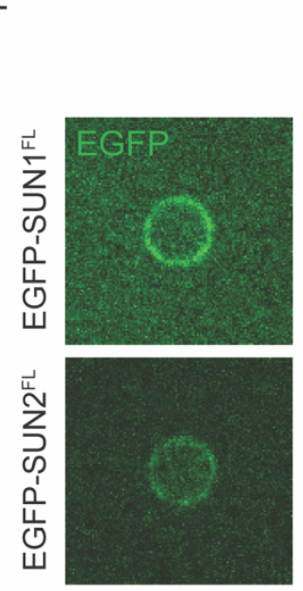

EGFP-SUN1 ${ }^{\mathrm{FL}}$
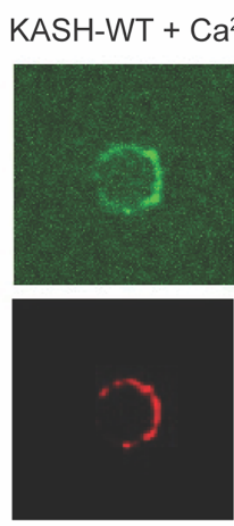

EGFP-SUN2 ${ }^{\mathrm{FL}}$

$\mathrm{KASH}-\mathrm{WT}+\mathrm{Ca}^{2+} \mathrm{KASH}-\mathrm{dPPPT}+\mathrm{Ca}^{2+}$
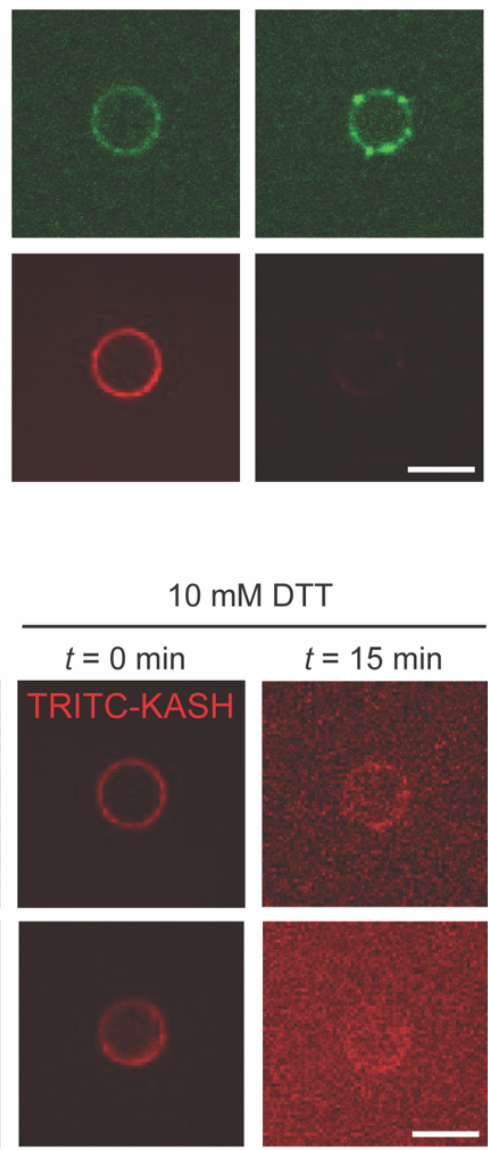

$\mathrm{B}$
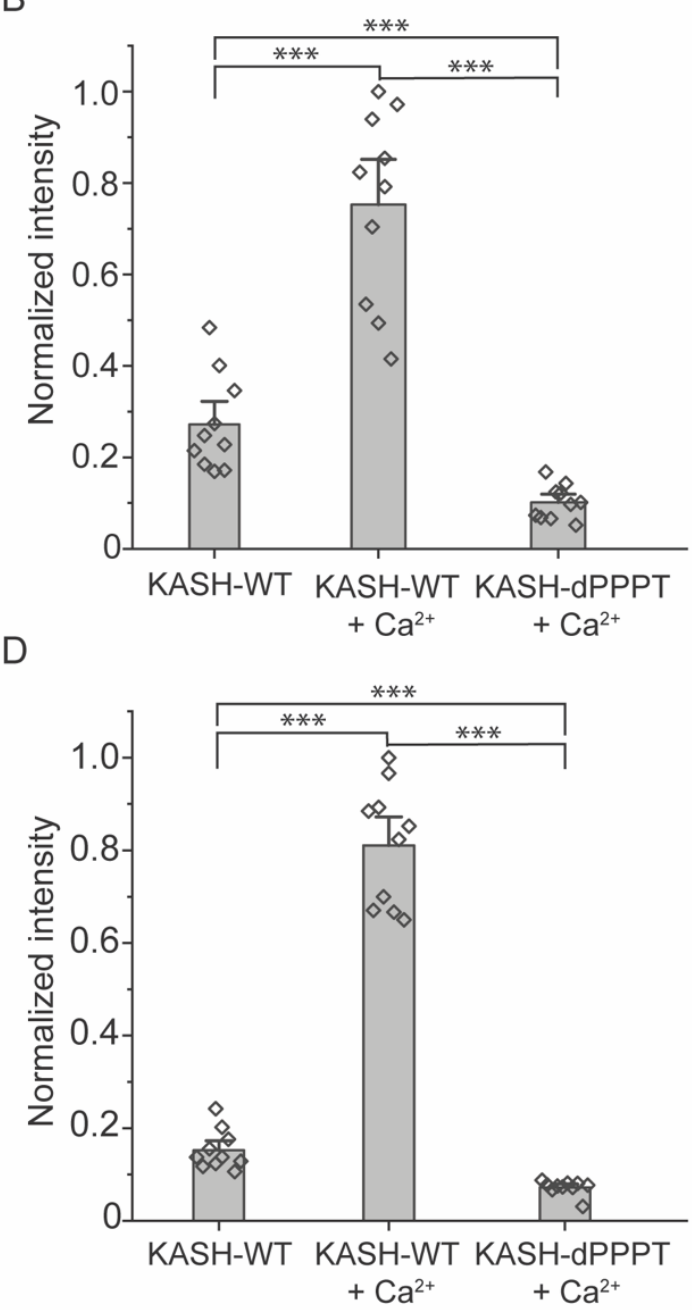

$\mathrm{F}$

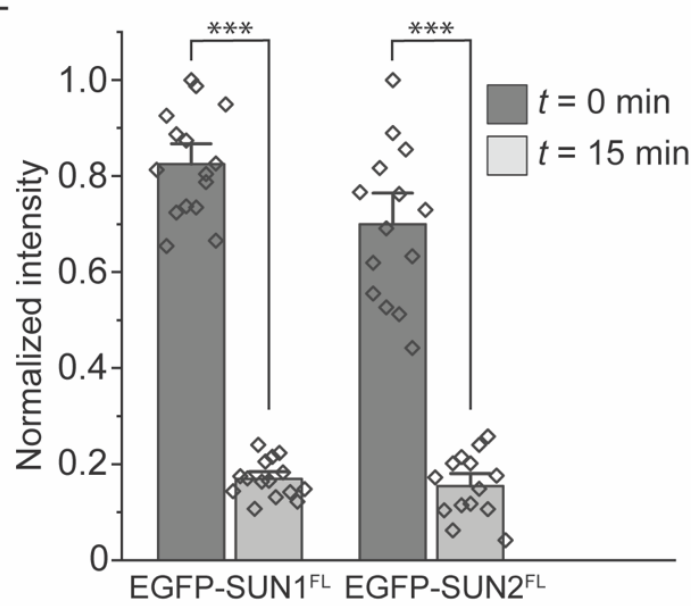

Figure 3: SUN-KASH interaction is enhanced by calcium and requires oxidizing conditions. $A$. Confocal fluorescence images of SUPER templates reconstituted with EGFP-SUN $1^{\mathrm{FL}}$ after incubation with TRITC-KASH ${ }^{\mathrm{WT}}$ or TRITC-KASH ${ }^{\mathrm{dPPPT}}$ peptides in the presence and absence of $1 \mathrm{mM}$ calcium. $\mathrm{B}$. Bar graph depicting the normalized intensity of KASH binding for the different conditions shown in $\mathrm{A}$. C. Confocal fluorescence images of SUPER templates reconstituted with EGFP-SUN2 ${ }^{\mathrm{FL}}$ after incubation with TRITC-KASH ${ }^{\mathrm{WT}}$ or TRITC-KASH ${ }^{\mathrm{dPPPT}}$ peptides in the presence and absence of $1 \mathrm{mM}$ calcium. D. Bar graph depicting the normalized intensity of KASH binding for the different conditions shown in C. and E. Confocal fluorescence images of TRITC-KASH ${ }^{\mathrm{WT}}$ on SUN protein-reconstituted SUPER templates following incubation with $10 \mathrm{mM}$ DTT. F. Bar graph of KASH binding before and after 
bioRxiv preprint doi: https://doi.org/10.1101/2021.04.11.439350; this version posted August 24, 2021. The copyright holder for this preprint (which was not certified by peer review) is the author/funder. All rights reserved. No reuse allowed without permission.

DTT addition. Scale bars: $5 \mu \mathrm{m}$. Error bars represent standard error of the mean. 10-15 beads were analyzed for each condition from $n=3$ experiments. ${ }^{* * *}$ denotes $p<0.001$ by pairwise Student's $t$-test. 
A
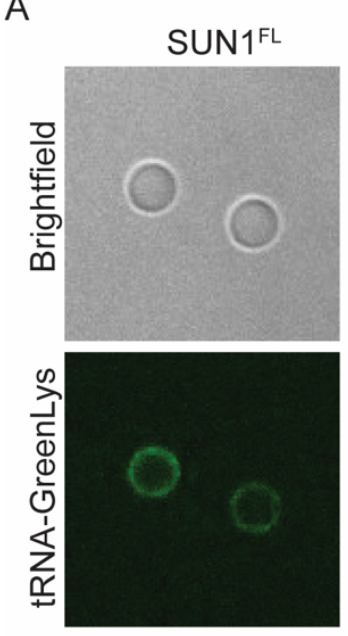

C
B
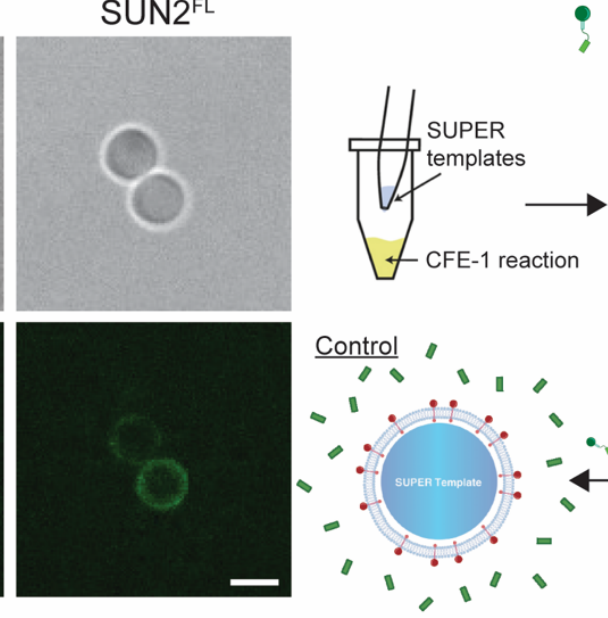

EGFP-SUN ${ }^{L D}$
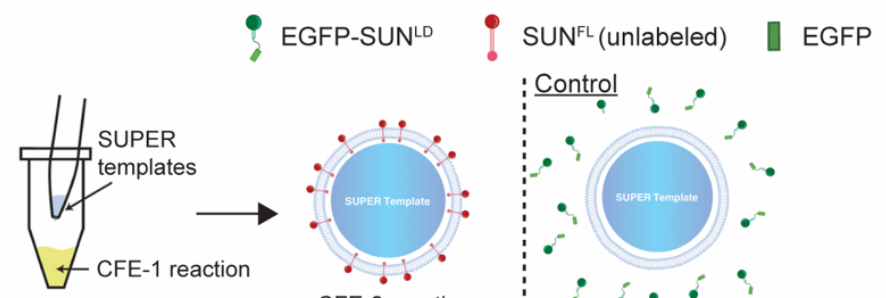

CFE-2 reaction
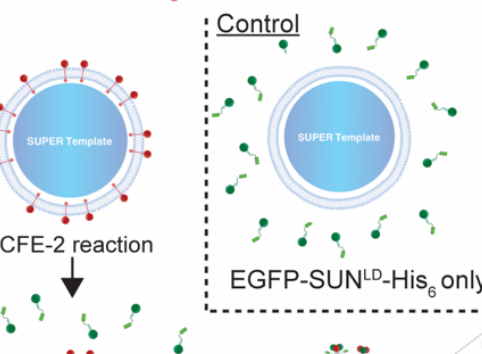

EGFP-SUN ${ }^{L-}$-His only

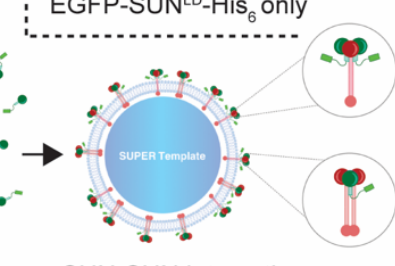

SUN-SUN interaction

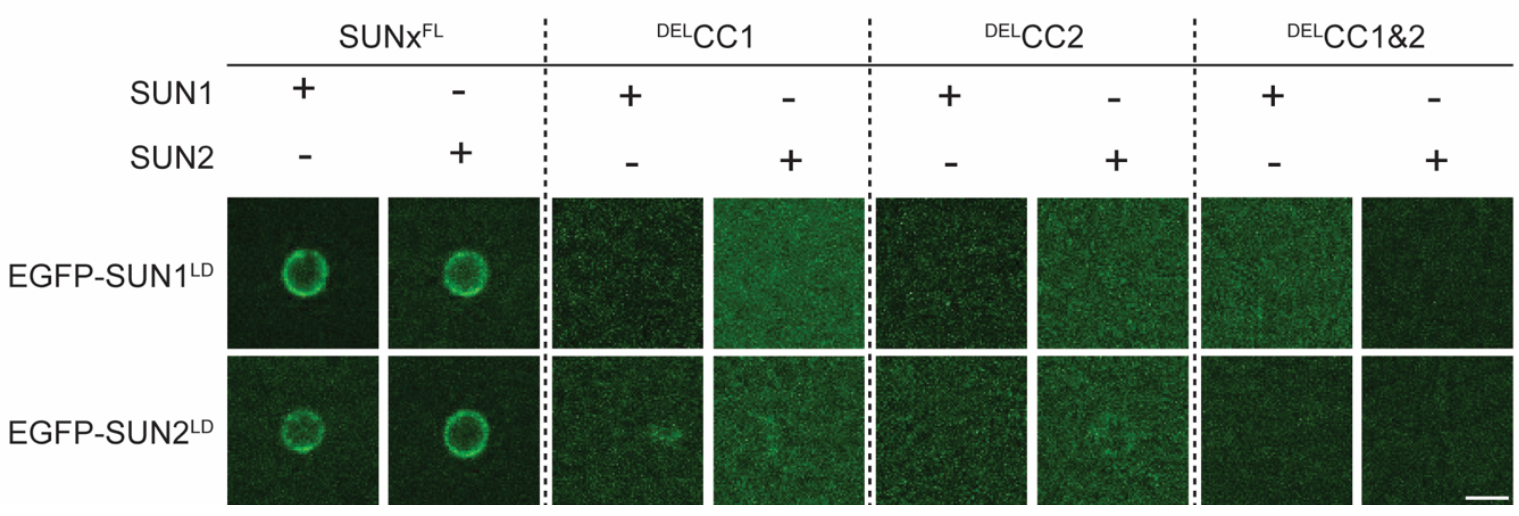

Figure 4: Coiled coil (CC) domains are important for SUN-SUN homomeric and heteromeric interactions. A. Confocal fluorescence images of full-length SUN proteins labelled with GreenLys during CFE reaction and reconstituted on SUPER templates. B. Schematic depicting the workflow for testing interaction between SUN proteins using sequential CFE-based reconstitution on SUPER templates. An unlabeled SUN protein is first expressed (full-length or a ${ }^{\mathrm{DEL}} \mathrm{CC}$ mutant) and reconstituted on SUPER templates, followed by a second CFE reaction that incorporates EGFP-tagged SUN1/2 ${ }^{\mathrm{LD}}$. Two controls are illustrated as well, one control with EGFP in the presence of full-length SUN proteins (bottom left) and another control with CFE of EGFP-tagged SUN1/2 ${ }^{\mathrm{LD}}$ in the absence of reconstituted full-length SUN proteins (top right) C. Test of SUN-SUN interactions of full-length SUN proteins and different coiled coil mutants carried out using the workflow in outlined in B. A panel of confocal fluorescence images reveals the interaction between the different SUN constructs. Experiments were repeated 3 times. Scale bars: $5 \mu \mathrm{m}$. 